\title{
Negative-Pressure Ventilation in Pediatric Acute Respiratory Failure
}

\author{
Amanda B Hassinger MD MSc, Ryan K Breuer MD, Kirsten Nutty NP, Chang-Xing Ma PhD, and \\ Omar S Al Ibrahim MD
}

\begin{abstract}
BACKGROUND: The objective of this work was to describe the use of negative-pressure ventilation (NPV) in a heterogeneous critically ill, pediatric population. METHODS: A retrospective chart review was conducted of all patients admitted to a pediatric ICU with acute respiratory failure supported with NPV from January 1, 2012 to May 15, 2015. RESULTS: Two hundred thirty-three subjects at a median age of $\mathbf{1 5 . 5}$ months were supported with NPV for various etiologies, most commonly bronchiolitis (70\%). Median (interquartile range) duration of support was 18.7 (8.7$34.3)$ h. The majority were NPV responders $(70 \%)$, defined as not needing escalation to any form of positive-pressure ventilation. In non-responders, escalation occurred at a median (interquartile range) of 6.9 (3.3-16.6) h. More NPV non-responders had upper-airway obstruction $(P=.02)$, and fewer had bronchiolitis $(P=.008)$ compared with responders. A bedside scoring system developed on these data was $98 \%$ specific in predicting NPV failure by $4 \mathrm{~h}$ after NPV start (area under the curve $0.759,95 \%$ CI $0.675-0.843, P<.001)$. Complications from NPV were rare (3\%); however, delayed enteral nutrition (33\%) and continuous intravenous sedation use $(51 \%)$ in children while receiving NPV were more frequent. The annual percentage of pediatric ICU admissions requiring intubation declined by $28 \%$ in the $3 \mathrm{y}$ after NPV introduction, compared with the $3 \mathrm{y}$ prior. CONCLUSIONS: NPV is a noninvasive respiratory support for pediatric acute respiratory failure from all causes with few complications and a $70 \%$ response rate. Children receiving NPV often required intravenous sedation for comfort, and one third received delayed enteral nutrition. Those who required escalation from NPV worsened within $6 \mathrm{~h}$; this may be predictable with a bedside scoring system. Key words: respiratory failure; children; negative-pressure ventilation; device safety; noninvasive ventilation; treatment efficacy. [Respir Care 2017;62(12):1540-1549. (C 2017 Daedalus Enterprises]
\end{abstract}

\section{Introduction}

Acute respiratory failure (ARF) is the most common reason for admission to a pediatric ICU in the United States. ${ }^{1}$ Patients with ARF are commonly managed with

\footnotetext{
Drs Hassinger, Breuer, and Al Ibrahim are affiliated with the Department of Pediatrics, Jacobs School of Medicine and Biomedical Sciences, University at Buffalo, Buffalo, New York. Ms Nutty is affiliated with the Division of Pediatric Critical Care, Women and Children's Hospital of Buffalo, Buffalo, New York. Dr Ma is affiliated with the Department of Biostatistics, School of Public Health and Health Professions University at Buffalo, Buffalo, New York.
}

The authors have disclosed no conflicts of interest.

Dr Patankar presented a version of this work at the Annual Congress of the Society of Critical Care Medicine, held January 21-25, 2017, in Honolulu, Hawaii. supraphysiologic oxygen, high rates of flow, and air pressure delivered through invasive and noninvasive modalities, all with inherent risks and clinical implications. ${ }^{2}$ Invasive positive-pressure ventilation is independently associated with increased lengths of stay and risk of bacterial pneumonia, requires intravenous sedation, and can precipitate lung injury. The damage from invasive ventilation is due to the delivery of positive pressure in a nonphysiologic way, causing barotrauma from excessive pressures, volutrauma from excessive tidal volumes, and atelectrauma from heterogeneous tidal volume delivery.1,3

\footnotetext{
Correspondence: Amanda B Hassinger MD MSc, Division of Pediatric Critical Care, Women and Children's Hospital of Buffalo, 219 Bryant Street, Buffalo, NY 14222.
}

DOI: $10.4187 /$ respcare. 05531 


\section{Negative-Pressure Ventilation in Pediatric ARF}

Noninvasive respiratory support modalities such as bilevel or continuous positive airway pressure (BPAP or CPAP) require tightly fitting masks that may not accommodate all-sized patients and can precipitate anxiety and make secretions or emesis problematic. Delivering extrathoracic negative pressure using cuirass ventilation without engaging the face or the airway is an alternative strategy that supports respiratory effort without the risks of invasive mechanical ventilation or NIV. Exploiting physiologic negative pressure in the place of positive pressure could avoid the dangers inherent in nonphysiologic ventilator support.

Negative-pressure ventilation (NPV), applied by an iron lung was the first mode of mechanical ventilation created to support respiratory failure due to poliovirus. It fell out of favor because of its cumbersome nature and its detrimental hemodynamic effects on venous return from the abdomen. Mechanical ventilation delivering positive pressure via the airway circumvented these issues and became the standard of care. Over time, as the morbidities associated with invasive positive-pressure ventilation were discovered, negative pressure technology was re-explored. ${ }^{4}$ Newer NPV devices deliver negative extrathoracic pressure to the chest wall through a plastic chest plate, or cuirass. These devices have the ability to provide continuous negative pressure to the chest, similar to CPAP. It also can be set in the control mode to deliver biphasic pressure, a negative pressure to augment inspiration and positive pressure to facilitate exhalation. Last, it can provide rapid alternating positive and negative pressure to the chest as physiotherapy to aid in secretion clearance, similar to cough assist. Because it does not rely on a face mask, NPV is suitable for patients with abnormal facial morphologies, anxiety, excessive oropharyngeal secretions, and emesis. ${ }^{5}$ In addition, current NPV devices augment right-ventricular preload and cardiac output, making it hemodynamically safer than previous NPV devices. ${ }^{6,7}$

To date, there have been few pediatric studies describing the use of NPV in children with ARF from all causes. A 2013 Cochrane review concluded that more studies are needed to assess NPV safety and outcomes after it found only one study comparing NPV with NIV in 33 children with bronchiolitis, published only in abstract form. ${ }^{8,9}$ Existing literature dedicated to NPV use is limited to small case series or case reports in homogeneous disease states, such as acute lung injury, neuromuscular disorders, ${ }^{7,10}$ neuromuscular disorders, ${ }^{11,12}$ central hypoventilation, ${ }^{4}$ bronchiolitis, ${ }^{8}$ heart failure, and postoperative management of congenital heart disease. ${ }^{6,13-15}$

No published data on complication rates of NPV in a general pediatric population are available. Reported complications and limitations of current NPV devices have included hypothermia, skin irritation at contact points, and upper-airway obstruction because tracheal pressures can become more negative during NPV than during spontane-

\section{QUICK LOOK}

\section{Current knowledge}

Acute respiratory failure in children is the most frequent indication for critical care in the United States. Noninvasive and invasive respiratory supports are available that provide positive-pressure support. Redesigned negative-pressure ventilation devices are available for children but have not been widely applied in heterogeneous pediatric populations.

\section{What this paper contributes to our knowledge}

In this large, single-center report, subjects age 2 months to $22 \mathrm{y}$ with acute respiratory failure from any cause were effectively supported with negative-pressure ventilation (NPV). Commonly used pressures and settings for NPV use in children were described. NPV led to clinical improvement in $70 \%$ of the children with a complication rate of $3 \%$. One third of children had a delay in enteral nutrition, and half were given continuous intravenous sedation while receiving NPV.

ous respiration. ${ }^{13}$ There have also been reports of gastroesophageal reflux and aspiration due to the effects of negative-pressure on the tone of the lower esophageal sphincter. ${ }^{16}$

We describe a single-center experience with NPV in pediatric subjects with acute respiratory failure from any cause admitted to a pediatric ICU. This study was performed to describe applicable patient populations, complication rates, technical aspects, and limitations of NPV use in a general, heterogeneous pediatric population with ARF.

\section{Methods}

This was a retrospective chart review of all patients with ARF admitted to the pediatric ICU supported by NPV from January 1, 2012 to May 15, 2015. Women and Children's Hospital of Buffalo has a 20-bed, multidisciplinary pediatric ICU that admits $1,200-1,300$ patients annually with medical and (non-cardiac) surgical diagnoses. The time frame for this study was chosen to capture the first 3 y during which NPV was in regular use at the study institution. The institutional review board of the University of Buffalo approved this study.

Initiation, titration, and duration of NPV use were at the discretion of the managing medical team because no protocol was in place. In general, NPV was started after subjects had ongoing distress, hypoxia, or hypercapnia despite maximal flows on high-flow nasal cannula (ie, $15-20 \mathrm{~L} / \mathrm{min}$ ). Starting and titrating NPV is similar to using NIV. Be- 


\section{Negative-Pressure Ventilation in Pediatric ARF}

cause CPAP would be the starting mode in NIV, continuous negative extrathoracic pressure is the starting mode most often in NPV. Subjects were escalated to control mode (akin to escalating from CPAP to BPAP) if they showed no clinical improvement on escalating continuous negative pressures. Control mode offers biphasic support with negative pressure to augment inspiration, alternating with positive pressure to provide an active expiratory phase, set at a mandatory rate that does not synchronize with patient effort (the synchronized mode was not approved for use in the United States during the study period). This allows patients to breathe with the NPV or between mandatory breaths provided by the NPV. When choosing control mode settings, the manufacturer recommends a 3:1 ratio of negative pressure amount to positive pressure amount, such as -12 to $+4 \mathrm{~cm} \mathrm{H}_{2} \mathrm{O}$, with a set respiratory frequency above the patient's intrinsic frequency, preferably $<60$ breaths/min. Subjects were fitted to the NPV using a plastic chest plate, or cuirass, that comes in 7 pediatric and 4 adult sizes. The cuirass can be reused, but disposable foam liners are individual-use-only and are essential to provide the seal between the cuirass and the negative pressure generated by the NPV device. Application is limited by cuirass fit, an issue with scoliosis, and machine availability.

The initial choice of NPV over other respiratory support modalities and the use of additional respiratory support while using NPV were also left to the discretion of the managing team based on the subject's clinical status and oxygen requirement; no protocol was in place. The study pediatric ICU routinely uses NIV as well as high-flow nasal cannula up to $20 \mathrm{~L} / \mathrm{min}$ and nasal intermittent positive-pressure support through the RAM cannula (Neotech, Valencia, CA). Physicians often choose between the RAM cannula and NPV in neonates with respiratory distress and between NIV and NPV in children 6 months of age and older. In the study pediatric ICU, types of noninvasive respiratory support other than NPV are used less frequently for non-invasive support of ARF than NPV due to the institutional experience and comfort with NPV; however, these data were not collected for the purposes of this study. On average, $7-8 \%$ of all children admitted to the study pediatric ICU require intubation and invasive mechanical ventilation for ARF from all causes. It is important to note that there were no introductions of new technology in the study pediatric ICU other than NPV during the study period.

\section{Subjects}

All subjects were children admitted to the pediatric ICU on or between the specified dates with ARF supported by NPV at any point during the admission. Acute respiratory failure was defined as the need for any level of respiratory support above high-flow nasal cannula, including invasive positive-pressure ventilation, NIV, and NPV. Patients were excluded if NPV was used for secretion clearance only or if NPV was attempted but not used.

\section{Data Collection}

Demographic data collected on all subjects included sex, age, weight, comorbidities, and primary diagnosis at pediatric ICU admission. The particulars of NPV use (mode, settings) and any concurrent respiratory support were also collected. Data pertinent to the immediate physiologic response to NPV that were collected were heart rate, respiratory frequency, pulse oximetry reading, blood gas data, and oxygen requirement at $1 \mathrm{~h}$ before and $1,4,8,12$, and $24 \mathrm{~h}$ after NPV initiation. Expected complications, including hypothermia (core temperature $<35^{\circ} \mathrm{C}$ ), skin breakdown at cuirass contact points, use of sedation, delayed enteral nutrition ( $>48 \mathrm{~h}$ after pediatric ICU admission), and gastroesophageal reflux while receiving NPV, were likewise noted for each subject. Because no formal surveillance was in place, gastroesophageal reflux was defined for the purposes of this study as any providers' documentation of a witnessed aspiration event, emesis, or feeding intolerance that occurred while the subject received enteral feeding while on NPV support.

Outcomes measured included length of stay (pediatric ICU and hospital), duration of NPV support, length of acute respiratory failure, and mortality. Length of acute respiratory failure was defined as the time from the start to stop of any form of higher than high flow nasal cannula, as initiated by the clinical status and managing medical team. NPV non-responders were defined as any subjects who were taken off of NPV and transitioned to any form of positive-pressure ventilation (invasive or noninvasive) for ongoing ARF not improved during NPV. Without a standardized scoring system or protocol, the bedside clinician made the decision to escalate based on subjective clinical assessments. Institutional billing data for the calendar years 2009-2015 were used to measure crude annual pediatric ICU intubation rates before and after the introduction of NPV.

\section{Statistical Analysis}

Descriptive statistics were performed to describe the study population, the particulars of NPV usage, and responders and non-responders. Student $t$ tests were used to compare parametric data, and Mann-Whitney U or KruskalWallis tests were used for non-parametric data after using Kolmogorov-Smirnov testing to determine normality. Chisquare testing compared proportions between groups. Spearman correlation coefficients were calculated to assess associations between continuous non-parametric vari- 


\section{Negative-Pressure Ventilation in Pediatric ARF}

ables. To assess for a difference between NPV responders and non-responders, a bivariate comparison of baseline physiologic parameters was performed. This analysis was then used in a general mixed model to compare the physiologic response to NPV in responders and non-responders over time. After preliminary data analysis uncovered an early time to NPV non-response, logistic regression models were used to generate a scoring system to predict the need for escalation of care at a clinically meaningful time point based on descriptive data. Area-under-the-curve analysis determined the predictive power of the score. All analyses were performed using IBM SPSS 22 (SPSS, Chicago, Illinois) and SAS (SAS Institute, Cary, North Carolina) with significance set at $P<.05$.

\section{Results}

\section{Study Population}

Over the study period, 4,145 patients were admitted to the pediatric ICU, 233 (5.6\%) of whom were supported with NPV for acute respiratory failure. Table 1 displays the demographic data of the study population. Bronchiolitis was the most common pediatric ICU admission diagnosis, occurring in $162(70 \%)$ of 233 subjects.

In most instances, NPV was not the first-line respiratory support; it was most often used after subjects were trialed on high-flow nasal cannula (79\%) and less often after noninvasive support, with the RAM cannula or CPAP $(8.6 \%)$. Five subjects were placed on NPV after extubation from invasive positive-pressure ventilation, with one of these 5 requiring re-intubation despite NPV use.

\section{Description of NPV Settings}

On initiation of NPV, most subjects (93.6\%) were placed on the continuous negative extrathoracic pressure mode before the control mode. Twenty-seven subjects (11.6\%) used the secretion clearance mode as well as continuous negative extrathoracic pressure or control, a strategy that did not vary in frequency from year to year $(P=.43)$.

In all subjects, NPV was started at a median (interquartile range $[\mathrm{IQR}])$ of $3.0(0.5-12.1) \mathrm{h}$ after pediatric ICU admission. Subjects placed on continuous negative extrathoracic pressure at initiation $(n=218)$ were started at an average pressure of $-12 \pm 2.5 \mathrm{~cm} \mathrm{H}_{2} \mathrm{O}$ with a range of -6 to $-24 \mathrm{~cm} \mathrm{H}_{2} \mathrm{O}$ with an average most negative pressure of $-13 \pm 3.7 \mathrm{~cm} \mathrm{H}_{2} \mathrm{O}$ (range -8 to $-24 \mathrm{~cm} \mathrm{H}_{2} \mathrm{O}$ ). When the control mode was used at NPV initiation, the average starting negative pressure was $-17 \pm 3.8 \mathrm{~cm} \mathrm{H}_{2} \mathrm{O}$ with a range of -12 to $-24 \mathrm{~cm} \mathrm{H}_{2} \mathrm{O}$, and the average positive pressure was $+6 \pm 1.4 \mathrm{~cm} \mathrm{H}_{2} \mathrm{O}$ with a range of +4 to $+8 \mathrm{~cm} \mathrm{H}_{2} \mathrm{O}$.
Table 1. Description of the Study Population of Subjects Supported With Negative Pressure Ventilation for Acute Respiratory Failure From Any Cause

\begin{tabular}{|c|c|}
\hline Characteristics & $\begin{array}{c}\text { Study } \\
\text { Population } \\
(N=233)\end{array}$ \\
\hline Age, median (IQR) months & $15.5(7.6-39.6)$ \\
\hline Weight, median (IQR) kg & $10.4(7.8-14)$ \\
\hline Male sex, $n(\%)$ & $136(58.4)$ \\
\hline \multicolumn{2}{|l|}{ Primary diagnosis, $n(\%)$} \\
\hline Bronchiolitis & $162(69.5)$ \\
\hline Status asthmaticus & $21(9)$ \\
\hline Other (eg, pneumonia) & $50(21.4)$ \\
\hline \multicolumn{2}{|l|}{ Preexisting comorbidities, $n(\%)$} \\
\hline Reactive airway disease & $114(48.9)$ \\
\hline Upper-airway obstruction & $18(7.7)$ \\
\hline Chronic lung disease & $36(15.5)$ \\
\hline Neuromuscular disorder & $15(6.4)$ \\
\hline \multicolumn{2}{|l|}{ Year of admission to PICU, $n(\%)$} \\
\hline 2012 & $13(5.6)$ \\
\hline 2013 & $57(24.5)$ \\
\hline 2014 & $119(51.1)$ \\
\hline 2015 & $44(18.9)$ \\
\hline Length of NPV use, median (IQR) h & $18.7(8.7-34.3)$ \\
\hline $\begin{array}{l}\text { Time to start of NPV after PICU admission, } \\
\text { median (IQR) h }\end{array}$ & $3.0(0.5-12.1)$ \\
\hline Length of mechanical ventilation, median (IQR) d & $1.4(0.72-3.3)$ \\
\hline Length of stay in the PICU, median (IQR) d & $3.5(2.3-6.8)$ \\
\hline Length of stay in the hospital, median (IQR) d & $5.2(3.6-9.5)$ \\
\hline $\begin{array}{l}\mathrm{IQR}=\text { interquartile range } \\
\mathrm{PICU}=\text { pediatric ICU } \\
\mathrm{NPV}=\text { negative pressure ventilator }\end{array}$ & \\
\hline
\end{tabular}

Most subjects (88.4\%) were receiving high-flow nasal cannula while receiving NPV, at a median (IQR) flow of $15(10-15) \mathrm{L} / \mathrm{min}$ or $1.5(0.97-2.1) \mathrm{L} / \mathrm{kg} / \mathrm{min}$. Noninvasive support (via RAM cannula or CPAP) was used concurrently with NPV in $10.3 \%$ of subjects. Subjects receiving NIV without the ability to synchronize remained on continuous negative extrathoracic pressure only to avoid asynchrony between respiratory supports. Subjects receiving NIV while receiving NPV had no significant difference in complication rate compared with those not receiving NIV $(P=.09)$.

\section{Description of NPV Non-Responders}

Of the 233 subjects in the study population, $163(70 \%)$ had resolution of acute respiratory failure while receiving NPV, 63 (27\%) required change in respiratory support to other forms of positive-pressure ventilation, and 58 nonresponders ( $92.1 \%$ of the non-responders) were intubated. Of the remaining 7 subjects, 5 (2.1\%) were removed from NPV before clinical improvement secondary to complica- 


\section{Negative-Pressure Ventilation in Pediatric ARF}

Table 2. Comparison of Responders, Those With Resolution of Acute Respiratory Failure While Receiving Negative-Pressure Ventilation, With Non-Responders, Those Who Required Further Escalation Despite Negative-Pressure Ventilation

\begin{tabular}{|c|c|c|c|}
\hline Variables & Non-Responders $(n=63)$ & Responders $(n=170)$ & $P$ \\
\hline Age, median (IQR) mo & $19.0(7.2-70)$ & $15.1(7.6-33.8)$ & .11 \\
\hline Male sex, $n(\%)$ & $33(52.4)$ & $98(60.1)$ & .29 \\
\hline Weight, median (IQR) kg & $12(8.1-17.8)$ & $10(7.9-13.8)$ & .055 \\
\hline Primary diagnosis, $n(\%)$ & & & $.01 *$ \\
\hline Bronchiolitis & $34(54)$ & $128(75.3)$ & \\
\hline Status asthmaticus & $8(12.7)$ & $13(7.6)$ & \\
\hline Other & $21(33.3)$ & $28(16.5)$ & \\
\hline \multicolumn{4}{|l|}{ Preexisting comorbidities, $n(\%)$} \\
\hline Reactive airway disease & $34(54)$ & $75(44)$ & .28 \\
\hline Upper-airway obstruction & $9(14.2)$ & $8(4.7)$ & .02 \\
\hline Chronic lung disease & $14(22.2)$ & $20(11.8)$ & .061 \\
\hline Neuromuscular disorder & $6(9.5)$ & $8(4.7)$ & .20 \\
\hline \multicolumn{4}{|l|}{ Respiratory support before NPV, $n(\%)$} \\
\hline None & $8(12.7)$ & $5(3.1)$ & $.02 *$ \\
\hline HFNC & $44(69.8)$ & $140(85.9)$ & \\
\hline BPAP & $3(4.8)$ & $6(3.7)$ & \\
\hline NIV with RAM cannula & $4(6.3)$ & $7(4.3)$ & \\
\hline Conventional mechanical ventilation & $1(1.6)$ & $4(2.5)$ & \\
\hline Face mask & $3(4.8)$ & $1(0.6)$ & \\
\hline \multicolumn{4}{|l|}{ Vital signs before start of $\mathrm{NPV}$, mean $\pm \mathrm{SD}$} \\
\hline Heart rate, beats/min & $150 \pm 26$ & $150 \pm 26$ & .81 \\
\hline Frequency, breaths/min & $51 \pm 19$ & $53 \pm 17$ & .28 \\
\hline $\mathrm{S}_{\mathrm{pO}_{2}}, \%$ & $96 \pm 4.9$ & $97 \pm 7$ & .35 \\
\hline $\mathrm{F}_{\mathrm{IO}_{2}}$ & $0.63 \pm 0.28$ & $0.55 \pm 0.25$ & .038 \\
\hline \multicolumn{4}{|l|}{ Differences in NPV support } \\
\hline NPV control mode used, $n(\%)$ & $27(42.9)$ & $35(21.5)$ & .001 \\
\hline Receiving HFNC while receiving NPV, $n(\%)$ & $51(81)$ & 149 (91.4) & .03 \\
\hline Receiving NRS while receiving NPV, $n(\%)$ & $13(20.6)$ & $10(6.2)$ & .001 \\
\hline Initial NPV pressure setting, median (IQR) $\mathrm{cm} \mathrm{H}_{2} \mathrm{O}$ & $-12(-10$ to -14$)$ & $-12(-10$ to -14$)$ & .060 \\
\hline Most negative NPV pressure, median (IQR) $\mathrm{cm} \mathrm{H}_{2} \mathrm{O}$ & $-14(-12$ to -21$)$ & $-12(-10$ to -14$)$ & $<.001$ \\
\hline Time to start of NPV after PICU admission, mean (IQR) h & $3.7(0.5-15.9)$ & $2.6(0.5-9.7)$ & .41 \\
\hline Duration of NPV use, mean (IQR) h & $6.9(3.3-16.6)$ & $22.5(14.5-41.5)$ & $<.001$ \\
\hline Duration of any mechanical ventilation, mean (IQR) h & $140.7(69.0-283.9)$ & $23.3(14.1-41.9)$ & $<.001$ \\
\hline $\begin{array}{l}\text { values were obtained using Mann-Whitney } \mathrm{U} \text { testing to compare medians acros } \\
{ }^{*} \text { Comparison of the distribution of subjects in all categories of primary diagnosis } \\
\text { IQR = interquartile range } \\
\text { NPV = negative-pressure ventilation } \\
\text { HFNC = high-flow nasal cannula } \\
\text { BPAP = biphasic positive airway pressure } \\
\text { RAM = RAM cannula or nasal intermittent positive airway pressure } \\
\text { NRS = noninvasive respiratory support such as bilevel positive airway pressure, } \\
\text { PICU = pediatric ICU }\end{array}$ & $\begin{array}{l}\text { hi-square testing to compare proporti } \\
\text { y support before negative-pressure ve }\end{array}$ & rical variables. & \\
\hline
\end{tabular}

tions, and $2(0.9 \%)$ were removed for purposes of transport.

Table 2 displays the baseline comparison between NPV non-responders and responders. Non-responders tended to be larger and were less likely to carry a diagnosis of bronchiolitis. A greater percentage of non-responders had a history of upper-airway obstruction. Of all clinical baseline variables obtained, only average $\mathrm{F}_{\mathrm{IO}_{2}}$ before NPV initiation was higher in those subjects who failed NPV $(0.63 \pm 0.28)$ than in those who responded $(0.55 \pm 0.25)$ $(P=.038)$.
After adjusting for the presence of upper-airway obstruction, bronchiolitis, respiratory support before $\mathrm{NPV}, \mathrm{F}_{\mathrm{IO}_{2}}$ needed before NPV, and age, only a diagnosis of bronchiolitis was independently protective against (adjusted odds ratio [OR] $0.42,95 \%$ CI $0.21-0.83, P=.01$ ) and the presence of a history of upper-airway obstruction (adjusted OR 2.84, 95\% CI 1.02-7.91, $P=.045$ ) was independently associated with increased odds of non-response to NPV.

There was no statistical difference in the mean (IQR) time to start of NPV after admission in responders when compared with non-responders, $2.6(0.5-9.7) \mathrm{h}$ and 3.7 
$(0.5-15.9) \mathrm{h}(P=.41)$. The median (IQR) time to NPV start in 2012 was $5.3(1.5-18.4) \mathrm{h}$, which tended to be statistically higher than the time to NPV start in 2015 of $1.0(0.3-5.8) \mathrm{h}(P=.055)$. There was a positive correlation between time to NPV start and time to enteral nutrition $\left(\mathrm{r}_{\mathrm{s}}=0.37, P<.001\right)$, duration of mechanical ventilation $\left(\mathrm{r}_{\mathrm{s}}=0.17, P=.01\right)$, hospital length of stay $\left(\mathrm{r}_{\mathrm{s}}=0.30, P<.001\right)$, and length of stay in the pediatric ICU $\left(\mathrm{r}_{\mathrm{s}}=0.33, P<.001\right)$ but not duration of NPV use $\left(\mathrm{r}_{\mathrm{s}}=0.13, P=.064\right)$.

Non-responders stayed a median of $4 \mathrm{~d}$ longer in the pediatric ICU and $6 \mathrm{~d}$ longer in the hospital and remained in respiratory failure for $5 \mathrm{~d}$ longer than those who responded to NPV support, all $P<.001$. All of the mortalities $(n=4)$ occurred in subjects who required escalation from NPV.

\section{Impact of Age and Bronchiolitis on NPV Response}

To further describe the differences in the subjects, the population was dichotomized to those younger or older than $2 \mathrm{y}$ of age. The younger subjects more often had bronchiolitis, $87 \%$ versus $37 \%$, and less often had reactive airways disease, $35 \%$ versus $75 \%$, or neuromuscular disorders, $1.3 \%$ versus $16 \%$ (all $P<.001$ ). Only $23 \%$ of those $<2$ y old were non-responders, which was less than the $35 \%$ of those 2 y old and older who did not respond to NPV $(P=.059)$. The odds of needing escalation from NPV were $44 \%$ lower in young subjects (OR $0.57,95 \%$ CI $0.31-1.03, P=.060$ ) than in those $\geq 2$ y old, but this difference was not significant. After adjusting for a diagnosis of bronchiolitis, the adjusted OR for non-response and age rose to 0.89 (95\% CI $0.44-1.81, P=.75$ ) and lost the trend toward significance. Having a diagnosis of bronchiolitis decreased the odds of needing escalation from NPV by $59 \%$ (adjusted OR $0.41,95 \%$ CI $0.20-0.83$, $P=.01)$ and remained independently significant even after adjusting for age.

\section{Early Physiologic Response to NPV}

To adjust for age, the proportional change in breathing frequency ( $f$ ) was calculated by taking the difference in the frequency from each time point and dividing this by the original frequency (eg. $[80-60] / 80=25 \%$ change). In responders, $f$ fell by an average of $11 \%$ within $1 \mathrm{~h}$ of NPV use, which was higher than the average $2 \%$ decrease in the $\mathrm{f}$ of non-responders $(P=.051)$. At $4 \mathrm{~h}$, responders remained an average of $12 \%$ below initial $\mathrm{f}$, whereas non-responders were only $1 \%$ below the average initial $\mathrm{f}(P=.08)$. At $8 \mathrm{~h}$, responders remained at $12 \%$ below the initial $\mathrm{f}$, whereas the average $\mathrm{f}$ in non-responders went $7 \%$ above the initial $\mathrm{f}$ before NPV was started $(P=.02)$ (Fig. 1).

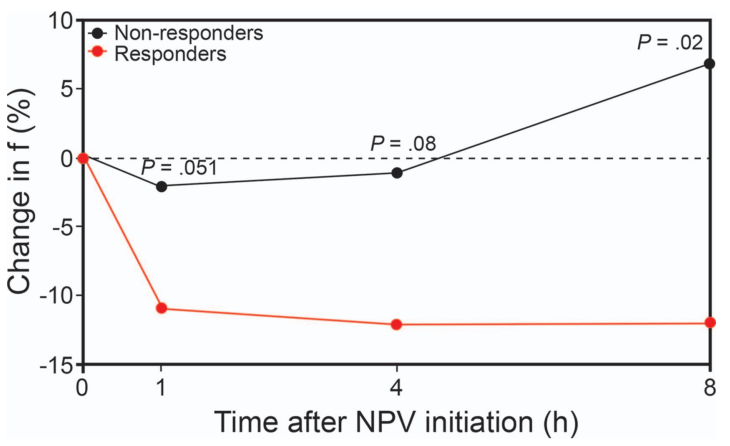

Fig. 1. Percent change in breathing frequency (f) from baseline in the first $8 \mathrm{~h}$ of negative pressure ventilation (NPV) therapy in responders versus non-responders.

In the general mixed model, there was no statistical difference in heart rate in responders compared with nonresponders over time because it was strongly correlated with the presence of a fever or concomitant albuterol administration. The only significant variable independently associated with response to NPV over time was $\mathrm{S}_{\mathrm{pO}_{2}}$. For every $5 \%$ increase in $\mathrm{S}_{\mathrm{pO}_{2}}$, the odds of responding to NPV increased by $1 \%$ (adjusted OR 1.01, 95\% CI 1.00-1.02, $P=.02)$.

Because non-responders required escalation of support at a median (IQR) of $6.9 \mathrm{~h}$ (3.3-16.6), all baseline physiologic variables and those collected at 1 and $4 \mathrm{~h}$ after NPV initiation were tested for inclusion in a scoring system intended to predict NPV non-response. The model with the most parsimonious number of variables and optimal specificity for non-response included the following variables: level of negative pressure at $4 \mathrm{~h}$, level of positive pressure at $4 \mathrm{~h}$, having bronchiolitis, and receiving NIV while receiving NPV. The area under the curve to predict NPV non-response with this model was 0.759 (95\% CI $0.675-0.843, P<.001$ ). Table 3 lists the coefficients for each variable used to calculate the score. A score of $\geq 0.5$ had $24 \%$ sensitivity and $98 \%$ specificity to predict NPV non-response, with a false negative rate of $17 \%$.

\section{Complications}

Expected complications from the cuirass were relatively rare and included hypothermia ( $n=2,0.9 \%$ of subjects), skin breakdown $(n=3,1.3 \%)$, and gastroesophageal reflux $(n=2,0.9 \%)$, leading to an overall complication rate of $3 \%$, or 7 of the 233 subjects. In 2 instances (both with skin breakdown), these complications resulted in cessation of NPV use. In subjects with these complications, the median (IQR) duration of NPV use was 23.3 (18.9-146.7) h compared with $18.5(8.3-34.2) \mathrm{h}$ in those without complications $(P=.052)$. 


\section{Negative-Pressure Ventilation in Pediatric ARF}

Table 3. Variable Coefficients in the Logistic Regression Model Used to Generate the Bedside Scoring System to Predict Failure to Respond to Negative-Pressure Ventilation

\begin{tabular}{lcc}
\hline \hline \multicolumn{1}{c}{ Variables } & Coefficients & Type of Variable \\
\hline Admission diagnosis of bronchiolitis & -0.9437 & Binary, $1=$ has bronchiolitis \\
Level of negative pressure used at $4 \mathrm{~h}$ & -0.3018 & Continuous, negative values \\
Level of positive pressure used at $4 \mathrm{~h}$ & -0.2730 & Continuous, positive values \\
Placed on NRS while receiving NPV & 1.8444 & Binary, $1=$ receiving NRS
\end{tabular}

An overall score of 0.5 has $98 \%$ specificity and $24 \%$ sensitivity for negative-pressure ventilation failure.

NRS $=$ noninvasive respiratory support (positive pressure), such as high-flow nasal cannula or CPAP

$\mathrm{NPV}=$ negative-pressure ventilation

Table 4. Change in Negative-Pressure Ventilation Settings Used and Subject Outcomes Including Negative-Pressure Ventilation Response and Complication Rates Over the Study Period

\begin{tabular}{|c|c|c|c|c|c|}
\hline Variables & $2012(n=13)$ & $2013(n=57)$ & $2014(n=119)$ & $2015(n=44)$ & $P$ \\
\hline $\begin{array}{l}\text { Time to NPV start after PICU admission, } \\
\text { median (IQR) h }\end{array}$ & $5.3(1.5-18.4)$ & $3.1(0.8-14.2)$ & $3.1(0.4-10.5)$ & $1.0(0.3-10.5)$ & $\begin{array}{c}.21(.055 \text { for } 2012 \\
\text { vs } 2015)\end{array}$ \\
\hline $\begin{array}{l}\text { Initial NPV pressure setting, median } \\
\text { (IQR) } \mathrm{cm} \mathrm{H}_{2} \mathrm{O}\end{array}$ & $-9(-8$ to -10$)$ & $-10(-10$ to -12$)$ & $-12(-10$ to -14$)$ & $-14(-12$ to -14$)$ & $<.001$ \\
\hline $\begin{array}{l}\text { Most negative pressure setting, median } \\
\text { (IQR) } \mathrm{cm} \mathrm{H}_{2} \mathrm{O} \text { ) }\end{array}$ & $-12(-8$ to -12$)$ & $-12(-10$ to -15$)$ & $-12(-12$ to -14$)$ & $-14(-12$ to -18$)$ & $<.001$ \\
\hline Duration of NPV use, median (IQR) h & $34.4(15.8-52.2)$ & $17.0(6.5-43.2)$ & $18.4(10.2-33.2)$ & $18.9(8.3-32.2)$ & .22 \\
\hline Complications, ${ }^{*} n(\%)$ & $1(7.7)$ & $2(3.5)$ & $1(0.8)$ & $3(6.8)$ & .16 \\
\hline Sedation given, $n(\%)$ & $2(15.4)$ & $17(29.8)$ & $61(51.3)$ & $38(86.4)$ & $<.001$ \\
\hline Delayed enteral nutrition, $n(\%) \dagger$ & $6(46.2)$ & $20(35.1)$ & $34(29.3)$ & $16(36.4)$ & .56 \\
\hline NPV non-response rate, $n(\%)$ & 30.8 & 29.8 & 25.2 & 27.3 & .83 \\
\hline PICU length of stay, median (IQR) d & $4.7(2.8-9.5)$ & $4.3(2.5-9.1)$ & $3.2(2.1-5.8)$ & $3.6(2.6-8.8)$ & .17 \\
\hline Mortality, $n(\%)$ & 0 & $1(1.8)$ & $3(2.5)$ & 0 & .69 \\
\hline \multicolumn{6}{|c|}{$\begin{array}{l}\text { Comparisons were made using Kruskal-Wallis or chi-square testing. } \\
* \text { Complications included are gastroesophageal reflux, skin breakdown, and hypothermia. } \\
\dagger \text { Enteral nutrition was delayed if delivered more than } 48 \mathrm{~h} \text { after pediatric ICU admission. } \\
\text { NPV = negative pressure ventilation } \\
\text { PICU = pediatric ICU } \\
\text { IQR = interquartile range }\end{array}$} \\
\hline
\end{tabular}

Slightly more than half $(50.6 \%)$ of the subjects were placed on intravenous sedation while receiving NPV, primarily dexmedetomidine ( $n=116$ of $118,98.3 \%)$. There was no difference in the mean (IQR) duration of NPV use in subjects who received sedation, $19.0(9.2-34.2) \mathrm{h}$, and those who did not receive sedation, $17.2(8.3-34.4) \mathrm{h}$ $(P=.67)$. The median (IQR) duration of dexmedetomidine use was $22(10-49) \mathrm{h}$ at an average peak dose of $0.46 \pm 0.3 \mu \mathrm{g} / \mathrm{kg} / \mathrm{h}$.

\section{Change in NPV Use and Outcomes Over the Study Period}

Table 4 displays the aspects of NPV use that changed over the study period. From 2012 to 2015, use of NPV and pressure settings became significantly more negative. Despite more negative pressures used in later years, the complication rates did not change over time, with the excep- tion of sedation use, which did increase from $15.4 \%$ of subjects receiving NPV in 2012 to $86.4 \%$ in 2015 $(P<.001)$. The percentage of subjects who failed NPV also did not change over the study period $(P=.83)$; nor did the median length of pediatric ICU stay for subjects receiving NPV $(P=.17)$.

As a non-subjective marker of the potential impact of widespread NPV use, the percentage of all pediatric ICU admissions placed on invasive positive-pressure ventilation each year was calculated before and after NPV introduction. From 2009 to 2011, an average of $9.9 \%$ of all pediatric ICU admissions underwent endotracheal intubation. This fell to $7.1 \%$ in $2012-2015$, an absolute rate reduction of $28 \%$ from the prior $3 \mathrm{y}$. This absolute difference did not reach statistical significance $(P=.15)$. There were no data collected related to the use of other noninvasive support modalities over this time to analyze this comparison further; however, as reported previously, no 


\section{Negative-Pressure Ventilation in Pediatric ARF}

other new technologies were added in routine patient care during this time frame other than NPV.

\section{Nutrition in Subjects While Receiving NPV}

The majority of the subjects (86\%) were kept nil per os while receiving NPV; $14(6 \%)$ received total parenteral nutrition. Enteral nutrition, when delivered, was given via nasogastric or gastrostomy tube $(n=13,5.6 \%)$, via nasoduodenal or jejunostomy tube $(n=11,4.7 \%)$, or by mouth $(n=9,3.9 \%)$. Two subjects experienced signs of gastroesophageal reflux, which included emesis, signs of reflux, or suspicion of aspiration as documented by providers. Receiving enteral nutrition while receiving NPV did increase the occurrence of gastroesophageal reflux events $(P=.001)$, but only in those fed via the duodenal route; no gastroesophageal reflux occurred in subjects fed by mouth or into the stomach $(P=.039$ for comparing rates of gastroesophageal reflux in those receiving gastric vs duodenal feeding).

Overall, enteral nutrition was delayed by $>48 \mathrm{~h}$ in 76 subjects $(32.6 \%)$. There was no difference in the proportion of responders and non-responders who had delays in enteral nutrition $(23.7 \%$ vs $27.3 \%, P=.41)$. However, subjects with a delay of enteral nutrition spent more time receiving NPV than those without a delay (median of $36.8 \mathrm{~h}$ vs $15.6 \mathrm{~h}, P<.001$ ). After adjusting for bronchiolitis, the need for NIV while receiving NPV, and the presence of a neuromuscular disorder, only age and duration of NPV use were independently associated with delayed enteral nutrition. Every hour receiving NPV increased the odds of delayed enteral nutrition by $3 \%$, adjusted OR 1.03 (95\% CI $1.02-1.05, P<.001)$. In addition, those with delayed enteral nutrition had extended periods of time before NPV was started after pediatric ICU admission, 9.6 (1.8-22.3) h versus $1.6(0.4-6.0) \mathrm{h}(P<.001)$, implying that tenuous or worsening clinical status could explain the delay in enteral nutrition.

\section{Discussion}

This study is the largest report of NPV use in a general pediatric critical care population to date. Intubation rates with other forms of noninvasive respiratory support (CPAP and BPAP) in pediatric acute respiratory failure and pediatric ARDS have been reported as high as $35-48 \%$ in studies attempting to validate predictive scoring systems similar to the one presented here ${ }^{17,18}$ or on par with our findings at $23 \%$ in 2 pediatric observational studies reporting the management of pediatric acute respiratory failure from all causes with other modalities of NIV in 114 and 150 children. ${ }^{19,20}$ Because the physiologic effects of NPV are not directly comparable with that of NIV, these rates cannot be directly compared, and our results are presented with the intent to inform use of a newer technology in children with heterogeneous disease states and cannot conclude on the efficacy of NPV. In this heterogeneous population, $70 \%$ of children with acute respiratory failure improved with NPV support and did not require further escalation of care.

The need for escalation of support happened early, emphasizing the need to monitor closely to avoid delay in invasive ventilation. We did not find an association between delays in NPV start and the need for intubation or escalation. In our experience, NPV is a suitable mode of support comparable with NIV that provides noninvasive lung recruitment while allowing secretion clearance support and the ability to suction. It is especially effective in young children with bronchiolitis and can be safe to use in conjunction with other modalities for noninvasive support.

Overall, expected complications from NPV were rare. A complication rate of $3 \%$ is far lower than the $6-8 \%$ of adults $^{21}$ and as many as $72 \%$ of children ${ }^{22}$ who have been reported to develop interface-related skin ulcers or skin compromise from NIV. The lower rate of interface skin irritation from NPV than NIV is probably due to the foam padding on the RTX cuirass, which allows for less irritation and moisture trapping than the plastic seal on NIV devices. ${ }^{22}$ The fact that more negative pressures were used without an increase in skin breakdown, hypothermia, or reflux events over the study period is important to report to any center considering adoption of NPV. The relationship between the duration of NPV use and increased prevalence of delayed enteral nutrition and a trend toward more expected complications implies that our complication rates could be falsely low due to the short overall duration that our subjects were receiving NPV. The performance of scheduled skin checks by the bedside nurse and respiratory therapist every $4 \mathrm{~h}$ during NPV in our experience provided adequate surveillance for skin irritation.

The prevalent use of sedation and delay in enteral nutrition in children receiving NPV are 2 key findings that provide important feedback to current and future users as potential limitations of NPV technology. The increase in sedation use over the study period probably reflects a change in institutional practice as opposed to a change in patient comfort, although the use of more negative pressures could have contributed to this need. A larger contributor was probably the effect of routine practice patterns as use of NPV increased. Providers automatically ordered sedation to ensure maximal cooperation and delivery of respiratory support without confirming that sedation was necessary. Because the respiratory side effects of dexmedetomidine are mild, there was little clinical objection to this practice. However, in hindsight, the peak of discomfort during NPV support is at initiation, when a single dose of sedative may be adequate and continuous infusions unnecessary, especially in continuous negative extrathoracic pressure mode. 


\section{Negative-Pressure Ventilation in Pediatric ARF}

Because this is not currently standard of care for other modalities of NIV, further research is warranted to determine sedation needs to provide optimal comfort and synchrony during delivery of NPV and to determine whether the risk of sedation is worth the benefit of NPV.

In addition, the tendency to avoid enteral feeding during NPV arose from previous reports of the risk of reflux and aspiration, especially in younger children. The rates reported here are low enough to provide support for feeding trials in children receiving NPV. If concerns of intubation were the primary motivation behind withholding enteral nutrition, our findings that subjects tended to fail early allow a window after which to consider starting feeding. Future prospective randomized trials could establish whether gastric or duodenal feeds are safest during NPV and in what age groups each might be more appropriate.

The scoring system developed from these data, although not validated, has the potential to assist clinical decision making in children with ARF managed with NPV. With high specificity, this tool could allow clinicians to prevent a delay in escalation in care for subjects who are more likely to fail NPV. This is a critical aspect to the use of noninvasive respiratory support, as illustrated in reports of increased mortality when NIV is used in ARDS and intubation is delayed.23,24 This scoring tool needs to be validated in a prospective manner before it is recommended for use.

The results related to time to NPV initiation more likely reflect the natural evolution of disease in viral illnesses that worsen over time rather than clinical delays in therapy. Evidence of this interpretation is further provided by the data showing that although NPV was started earlier in the later years of the study as institutional comfort grew, there was no change in the proportion of subjects who did not respond to NPV. Nevertheless, future research into the optimal timing of the application of NPV is needed and should be tailored to each disease state, since bronchiolitis is unlikely to be comparable with pneumonia in an adolescent with neuromuscular disease.

As a single-center, retrospective study, there were limitations. These results are intended only to describe practice patterns, complications, and patient outcomes in children with ARF managed with the Hayek RTX, a device not found in many pediatric critical care centers. The conclusions drawn are not intended to extend beyond the strength of the data, only to describe our experience. There is probably a selection bias impacting the measure of NPV efficacy in this cohort, as evidenced by the short duration of NPV use, the young age of our population, and the large proportion of those with bronchiolitis. The benefit of NPV alone cannot be concluded from these results, especially when other modalities of noninvasive support were used in conjunction with NPV in $10 \%$ of these subjects. The changes in crude intubation rates were presented as an observation that we cannot attribute to the introduction of any other technology or respiratory support in our center. The power of our data cannot confirm an association with fewer intubations and widespread NPV use.

\section{Conclusions}

NPV is a noninvasive respiratory support for children with acute respiratory failure from varying etiologies that has few complications and a $70 \%$ response rate in a general pediatric population. Failure to respond to NPV may be predictable early after NPV initiation to allow informed clinical decision making about starting enteral feeding or proceeding to intubation. Sedation and feeding protocols are needed for critically ill children during NPV.

\section{REFERENCES}

1. Fuchs H, Nicolai T, Schmid MB, Krüger M. [Current concepts of weaning children from invasive ventilation]. Anasthesiol Intensivmed Notfallmed Schmerzther 2013;48(10):622-625.

2. Zielińska M, Zieliński S, Sniatkowska-Bartkowska A. Mechanical ventilation in children: problems and issues. Adv Clin Exp Med 2014;23(5):843-848.

3. Turner DA, Arnold JH. Insights in pediatric ventilation: timing of intubation, ventilatory strategies, and weaning. Curr Opin Crit Care 2007;13(1):57-63.

4. Hartmann H, Jawad MH, Noyes J, Samuels MP, Southall DP. Negative extrathoracic pressure ventilation in central hypoventilation syndrome. Arch Dis Child 1994;70(5):418-423.

5. Corrado A, Gorini M. Negative-pressure ventilation: is there still a role? Eur Respir J 2002;20(1):187-197.

6. Shime N, Toida C, Itoi T, Hashimoto S. Continuous negative extrathoracic pressure in children after congenital heart surgery. Crit Care Resusc 2006;8(4):297-301.

7. Borelli M, Benini A, Denkewitz T, Acciaro C, Foti G, Pesenti A. Effects of continuous negative extrathoracic pressure versus positive end-expiratory pressure in acute lung injury patients. Crit Care Med 1998;26(6): 1025-1031.

8. Hartman H, Noyes, JP, Wright T, Wheatley R, Spencer A, Boon A. Continuous negative extrathoracic pressure ventilation in infants with bronchiolitis. Eur Respir J 1994;7(Supplement 18):379.

9. Shah PS, Ohlsson A, Shah JP. Continuous negative extrathoracic pressure or continuous positive airway pressure compared to conventional ventilation for acute hypoxaemic respiratory failure in children. Cochrane Database Syst Rev 2013;(11):CD003699.

10. Klonin H, Bowman B, Peters M, Raffeeq P, Durward A, Bohn DJ, et al. Negative pressure ventilation via chest cuirass to decrease ventilator-associated complications in infants with acute respiratory failure: a case series. Respir Care 2000;45(5):486-490.

11. Hillberg RE, Johnson DC. Noninvasive ventilation. N Engl J Med 1997;337(24):1746-1752.

12. Shneerson JM, Simonds AK. Noninvasive ventilation for chest wall and neuromuscular disorders. Eur Respir J 2002;20(2):480-487.

13. Deep A, De Munter C, Desai A. Negative pressure ventilation in pediatric critical care setting. Indian J Pediatr 2007;74(5):483-488.

14. Toida C, Shime N, Itoi T, Yamagishi M. Recovery from Fontan circulation failure by application of continuous negative extrathoracic pressure. J Anesth 2007;21(2):282-284. 


\section{Negative-Pressure Ventilation in Pediatric ARF}

15. Deshpande SR, Kirshbom PM, Maher KO. Negative pressure ventilation as a therapy for post-operative complications in a patient with single ventricle physiology. Heart Lung Circ 2011;20(12):763765 .

16. Marino WD, Pitchumoni CS. Reversal of negative pressure ventilation-induced lower esophageal sphincter dysfunction with metoclopramide. Am J Gastroenterol 1992;87(2):190-194.

17. Agarwal R, Aggarwal AN, Gupta D. Role of noninvasive ventilation in acute lung injury/acute respiratory distress syndrome: a proportion meta-analysis. Respir Care 2010;55(12):1653-1660.

18. Duan J, Han X, Bai L, Zhou L, Huang S. Assessment of heart rate, acidosis, consciousness, oxygenation, and respiratory rate to predict noninvasive ventilation failure in hypoxemic patients. Intensive Care Med 2017;43(2):192-199.

19. Abadesso C, Nunes P, Silvestre C, Matias E, Loureiro H, Almeida H. Non-invasive ventilation in acute respiratory failure in children. Pediatr Rep 2012;4(2):e16.
20. Essouri S, Chevret L, Durand P, Haas V, Fauroux B, Devictor D. Noninvasive positive pressure ventilation: five years of experience in a pediatric intensive care unit. Pediatr Crit Care Med 2006;7(4):329-334.

21. Carron M. A new horizon for the use of non-invasive ventilation in patients with acute respiratory distress syndrome. Ann Transl Med 2016;4(18):348.

22. Visscher MO, White CC, Jones JM, Cahill T, Jones DC, Pan BS. Face masks for noninvasive ventilation: fit, excess skin hydration, and pressure ulcers. Respir Care 2015;60(11):1536-1547.

23. Cabrini L, Landoni G, Oriani A, Plumari VP, Nobile L, Greco M, et al. Noninvasive ventilation and survival in acute care settings: a comprehensive systematic review and metaanalysis of randomized controlled trials. Crit Care Med 2015;43(4):880-888.

24. Bellani G, Laffey JG, Pham T, Madotto F, Fan E, Brochard L, et al. Non-invasive ventilation of patients with ARDS: insights from the LUNG SAFE study. Am J Respir Crit Care Med 2017;195(1):67-77.

This article is approved for Continuing Respiratory Care Education credit. For information and to obtain your CRCE

(free to AARC members) visit

www.rcjournal.com 